\title{
Chiral dynamics and baryon resonances
}

\author{
Tetsuo Hyodo ${ }^{\text {a }}$ \\ ${ }^{a}$ Department of Physics, Tokyo Institute of Technology, Meguro 152-8551, Japan
}

\begin{abstract}
The structure of baryon resonance in coupled-channel meson-baryon scattering is studied from the viewpoint of chiral dynamics. The meson-baryon scattering amplitude can be successfully described together with the properties of the resonance in the scattering, by implementing the unitarity condition for the amplitude whose low energy structure is constrained by chiral theorem. Recently, there have been a major progress in the study of the structure of the resonance in chiral dynamics. We review the methods to clarify the structure of the resonance by focusing on the $\Lambda(1405)$ resonance.
\end{abstract}

\section{INTRODUCTION}

Chiral symmetry is the one of the guiding principles to study the low energy hadron physics, since it dictates the dynamics of the Nambu-Goldstone bosons with hadrons through the chiral low energy theorem. One of the great successes is the celebrated Weinberg-Tomozawa theorem [1,2], which determines the $\pi N$ and $\pi \pi$ scattering lengths in good agreement with experimental data. The current algebra technique was then refined by the establishment of power counting [3], which enables one to sort out the effective chiral Lagrangian and amplitude, leading to the systematic computation of the higher order corrections in chiral perturbation theory [4]. In recent years, the framework of chiral dynamics is extended to the resonance energy region, with the implementation of the coupled-channel unitarity condition of the scattering amplitude. This chiral coupled-channel approach has been providing fairly successful description of baryon resonances in meson octet-baryon octet scattering $[5,6,7,8]$.

Within the phenomenologically successful framework of meson-baryon scattering, it is interesting to study the structure of the resonances, which reflects the nonperturbative dynamics of strong interaction. In general, several constructions are possible for a baryon resonance, such as three-quark state, meson-baryon molecule, and more complicated structures. Physical state may 
be a superposition of all possible components with given quantum numbers, but the clarification of the dominant component should help our intuitive understanding of the resonance structure.

Among many resonances, the $\Lambda(1405)$ attracts considerable attention from several aspects. There is a long-standing discussion about its internal structure; whether it is a conventional three-quark state in quark models [9], or it is composed of meson-baryon molecule [10]. In a recent context, the properties of the $\Lambda(1405)$ is extensively discussed in relation with the possible kaonic nuclei [11]. Since the $\Lambda(1405)$ lies just below the $\bar{K} N$ threshold, it is considered to be a quasi-bound state of $\bar{K} N$ system, and thus it determines the bare interaction between kaon and nucleon. Indeed, it is found that the $\Lambda(1405)$ plays an important role in the extrapolation of the $\bar{K} N$ interaction down to far subthreshold energy region $[12,13,14]$. Experimental studies are also ongoing, in order to extract the unconventional nature of the $\Lambda(1405)$ resonance [15]. In this article, we present a series of recent theoretical works on the structure of the $\Lambda(1405)$ resonance in chiral dynamics.

\section{FRAMEWORK OF CHIRAL DYNAMICS}

Based on the N/D method [7], we write the general amplitude of $s$-wave mesonbaryon scattering at total energy $\sqrt{s}$ :

$$
T(\sqrt{s})=\frac{1}{V^{-1}(\sqrt{s})-G(\sqrt{s} ; a)}
$$

where $V(\sqrt{s})$ is the kernel interaction constrained by chiral symmetry, which is given in the matrix form with coupled-channel indices. The function $G(\sqrt{s} ; a)$ is the diagonal matrix with once subtracted dispersion integrals of the twobody phase-space function. For the $s$-wave two-body scattering, $G(\sqrt{s} ; a)$ contains one subtraction constant $a$ for each channel. Identifying the dispersion integral $G(\sqrt{s} ; a)$ as the loop function with dimensional regularization, Eq. (1) is regarded as the solution of the algebraic Bethe-Salpeter equation and the subtraction constant a plays a similar role with the cutoff parameter in the loop function. The interaction kernel $V(\sqrt{s})$ is determined by the order by order matching with the amplitude in chiral perturbation theory. At leading order, $V(\sqrt{s})$ is given by the Weinberg-Tomozawa (WT) term

$$
V_{i j}(\sqrt{s})=-\frac{C_{i j}}{4 f^{2}}\left(2 \sqrt{s}-M_{i}-M_{j}\right)
$$

where $C_{i j}, M_{i}$ and $f$ are the group theoretical factor, the mass of the baryon in channel $i$, and the meson decay constant, respectively. With the leading or-

der WT term, this framework is almost equivalent to the old coupled-channel 
works with vector meson exchange potential [10]. Based on chiral perturbation theory, it is now possible to include higher order corrections systematically $[5,8]$.

This approach has been successfully applied to the $S=-1$ and $I=0$ meson-baryon scattering, in which the $\Lambda(1405)$ resonance appears below the $\bar{K} N$ threshold. Total cross sections of the $K^{-} p$ scattering in different final states, threshold branching ratios, and the mass spectrum of the $\Lambda(1405)$ resonance are well reproduced $[5,6,7,8]$. Thanks to the universal properties of the Weinberg-Tomozawa theorem, the framework of chiral dynamics can be applied to many different hadron scattering systems, using the same mathematical machinery [16,17]. The successful applications to many resonances indicates the power of the chiral dynamics for the description of the hadron scattering.

\section{STRUCTURE OF BARYON RESONANCE}

\subsection{Origin of the resonance in the natural renormalization scheme}

In chiral dynamics, the excited baryons are described as resonances in the meson-baryon scattering amplitude. In this case, any components beyond the model space of two-body states (meson-baryon molecule) are expressed by the Castillejo-Dalitz-Dyson (CDD) pole contribution [18]. Therefore, to know the importance of the dynamical component, we should extract the CDD pole contribution in a transparent manner.

In Ref. [19], it is shown that the loop function $G$ can contain the CDD pole contribution, in addition to those in the interaction kernel $V$. In order to visualize all the CDD pole contribution in the kernel $V$, the natural renormalization scheme has been proposed. In this renormalization scheme, the subtraction constant is determined by purely theoretical argument, excluding the CDD pole contribution from the loop function. It is demonstrated that the deviation of the subtraction constant from the natural value can be expressed by the pole term in the effective interaction kernel. This pole term is interpreted as the seed of the resonance in the full amplitude. In this way, the natural renormalization scheme, together with the phenomenological amplitude, enables us to investigate the origin of the resonances in chiral dynamics.

Using this technique, we analyze the $\Lambda(1405)$ resonance in the $\bar{K} N$ scattering and the $N(1535)$ resonance in the $\pi N$ scattering. It is found that the $\Lambda(1405)$ resonance can be constructed mostly by the dynamical component, while the $N(1535)$ resonance require some CDD pole contribution on top of 
the dynamical meson-baryon components.

\subsection{Scaling analysis of number of color}

A novel method to investigate the quark structure of the resonances in chiral dynamics has been developed by using the $N_{c}$ scaling for the mesonic resonances [20]. Introducing the $N_{c}$ dependence through the particle masses and the coupling constants, the response of the resonance pole position against the variation of $N_{c}$ can be compared with the general $N_{c}$ scaling low of the mass and width of a $\bar{q} q$ meson, in order to estimate the size of the $\bar{q} q$ component in the resonance.

In Refs. [21,22], the baron resonances $\Lambda(1405)$ and $\Lambda(1670)$ have been studied in the same method. One non-trivial issue in the baryonic sector is the $N_{c}$ dependence of the coupling strength $C_{i j}$ in the leading order WT term $[16,17]$, which leads to the existence of the bound state in the large $N_{c}$ limit. For other parameters such as hadron masses and meson decay constant, we adopt the standard $N_{c}$ dependence derived from the general argument. In this way we obtain the meson-baryon scattering amplitude as a function of $N_{c}$.

General $N_{c}$ scaling for the excited baryon $R$ with $N_{c}$ quarks is given as $M_{R}=$ $\mathcal{O}\left(N_{c}\right)$ and $\Gamma_{R}=\mathcal{O}(1)$. We calculate the pole positions corresponding to the $\Lambda(1405)$ and $\Lambda(1670)$ with different values of $N_{c}$. When we change the $N_{c}$ from 3 to 12 , the imaginary parts of the pole positions for the $\Lambda(1405)$ and $\Lambda(1670)$ change drastically, in contrast to the scaling low of $q^{N_{c}}$ state, $\Gamma_{R}=\mathcal{O}(1)$. This result indicates that the three-quark component (at $N_{c}=3$ ) in the $\Lambda(1405)$ and $\Lambda(1670)$ should be small.

\subsection{Evaluation of the electromagnetic size}

A standard method to investigate the structure of a particle is to use the (virtual) photon probe. The form factor contains the information of the electromagnetic structure of the particle. In chiral dynamics, the resonance form factor can be calculated by introducing an external photon field. Since the resonance is expressed microscopically by the bubble sum of the meson-baryon loops, the photon field should be attached to the constituent mesons, baryons, and vertices.

In Ref. [23], the electromagnetic mean squared radii of the $\Lambda(1405)$ resonance is studied in chiral dynamics. Result of the charge radii of the $\Lambda(1405)$ shows a larger value than the ground state neutron. This implies that the charge distribution of the $\Lambda(1405)$ is much wider than the neutron in spatial size. 
This is consistent with the meson-baryon molecule picture, rather than the compact three-quark structure.

\section{SUMMARY}

We have discussed that the chiral dynamics describes the baryon resonances in meson-baryon scattering quite well, and the investigation of the structure of the resonance is now becoming available. By taking an example of the $\Lambda(1405)$, we show three approaches to reveal the structure of the resonance: the natural renormalization scheme, the $N_{c}$ scaling method, and the evaluation of the electromagnetic size. In view of these three independent analyses, we conclude that the $\Lambda(1405)$ resonance is mostly dominated by the meson-baryon dynamical component.

This conclusion may sound as a trivial result by the construction of the model, but it is not always the case. For instance, it has been found that the $N(1535)$ resonance and the $\rho$ meson contain substantial contributions of the non-dynamical components, although they appear as poles in the scattering amplitude, in the same way with the $\Lambda(1405)$. Therefore, the dynamical nature for the $\Lambda(1405)$ is a nontrivial consequence in chiral dynamics. On the other hand, the present analyses clarify the dominant structure only in a qualitative manner. More quantitative decomposition of several contributions will be highly appreciated. At the same time, the analysis of the three-quark like resonance, such as $\Delta(1232)$, provides a useful test of the method for the structure study. The study of the nontrivial structure of hadron resonances along this line will shed new light on the nonperturbative dynamics of low energy QCD.

\section{ACKNOWLEDGEMENTS}

The author is grateful to Atsushi Hosaka, Daisuke Jido, Luis Roca, and Takayasu Sekihara for fruitful collaborations. He thanks Wolfram Weise for kind invitation to the wonderful symposium. This work is partly supported by the Global Center of Excellence Program by MEXT, Japan through the Nanoscience and Quantum Physics Project of the Tokyo Institute of Technology.

\section{References}

[1] S. Weinberg, Phys. Rev. Lett. 17 (1966) 616. 
[2] Y. Tomozawa, Nuovo Cim. 46A (1966) 707.

[3] S. Weinberg, Physica A96 (1979) 327.

[4] J. Gasser and H. Leutwyler, Nucl. Phys. B250 (1985) 465.

[5] N. Kaiser, P.B. Siegel and W. Weise, Nucl. Phys. A594 (1995) 325.

[6] E. Oset and A. Ramos, Nucl. Phys. A635 (1998) 99.

[7] J.A. Oller and U.G. Meissner, Phys. Lett. B500 (2001) 263.

[8] M.F.M. Lutz and E.E. Kolomeitsev, Nucl. Phys. A700 (2002) 193.

[9] N. Isgur and G. Karl, Phys. Rev. D18 (1978) 4187.

[10] R.H. Dalitz, T.C. Wong and G. Rajasekaran, Phys. Rev. 153 (1967) 1617.

[11] Y. Akaishi and T. Yamazaki, Phys. Rev. C65 (2002) 044005.

[12] T. Hyodo and W. Weise, Phys. Rev. C77 (2008) 035204.

[13] A. Dote, T. Hyodo and W. Weise, Nucl. Phys. A804 (2008) 197.

[14] A. Dote, T. Hyodo and W. Weise, Phys. Rev. C79 (2009) 014003.

[15] M. Niiyama et al., Phys. Rev. C78 (2008) 035202.

[16] T. Hyodo, D. Jido and A. Hosaka, Phys. Rev. Lett. 97 (2006) 192002.

[17] T. Hyodo, D. Jido and A. Hosaka, Phys. Rev. D75 (2007) 034002.

[18] L. Castillejo, R.H. Dalitz and F.J. Dyson, Phys. Rev. 101 (1956) 453.

[19] T. Hyodo, D. Jido and A. Hosaka, Phys. Rev. C78 (2008) 025203.

[20] J.R. Pelaez, Phys. Rev. Lett. 92 (2004) 102001.

[21] T. Hyodo, D. Jido and L. Roca, Phys. Rev. D77 (2008) 056010.

[22] L. Roca, T. Hyodo and D. Jido, Nucl. Phys. A809 (2008) 65.

[23] T. Sekihara, T. Hyodo and D. Jido, Phys. Lett. B669 (2008) 133. 